This is the accepted manuscript of an article published by Taylor \& Francis in Popular

Music \& Society on March 10, 2015, available online:

http://www.tandfonline.com/doi/abs/10.1080/03007766.2015.1021174

\title{
The Playlist Experience: Personal Playlists in Music Streaming Services Anja Nylund Hagen
}

\section{Abstract}

Music streaming services encompass features that enable the organization of music into playlists. This article inquires how users describe and make sense of practices and experiences of creating, curating, maintaining, and using personal playlists. The analysis relies on a mixed-method study, including music-diary self-reports, online observations, and in-depth interviews with 12 heavy users of Spotify or/and WiMP Music. The findings suggest heterogeneous management of static and dynamic playlists based on structural and contextual schemes of aggregating music. User control motivates different playlist practices that demonstrate new ways of collecting music via streaming services but also derive from pre-digital collecting.

\section{Introduction}

Is it wrong, wanting to be at home with your record collection? It's not like collecting records is like collecting stamps, or beermats, or antique thimbles. There's a whole world in here, a nicer, dirtier, more violent, more peaceful, more colorful, sleazier, more dangerous, more loving world than the world I live in; there is history, and geography, and poetry, and countless other things I should have studied at school, including music. (Hornby 83)

In the novel High Fidelity, Nick Hornby describes the contents of a record collection as more powerful (or at least more interesting) than real life. Originality governs the acquisition, combination, and organization of one's records, and is the reason for the solace they provide. In contemporary Norway, where this study has been conducted, music-streaming services have supplanted records and CDs, as well as other digital music formats, to become the mainstream technology for everyday music listening. Norway is a leading international market when it comes to music streaming. In 2013 music-streaming revenues accounted for $75 \%$ of all recorded music revenues in Norway (Ifpi Norge), and seven out of ten Internet users accessed one of the two major services, Spotify and WiMP Music (TNS Gallup). Both these services are able to supply more than 20 million tracks - a truly extensive range of music that is available to both average listeners and hardcore fans.

In this atmosphere of music abundance, listening and collecting are in flux. People listen to more artists than ever before (Maasø) using streaming technology on their personal mobile devices. This service model transforms Hornby's obviously very possessive sense of music ownership into the relatively carefree, even whimsical status of the renter of access to vast musical archives via online subscription. As a renter, one assembles and maintains a personal playlist (or one chooses among playlists that are available for subscription from other users or the service provider). 
By looking at practices and experiences related to these personal playlists, this article investigates how people still manage to "collect" music in the age of the streaming service. I begin with the following research question: How do streaming users describe and make sense of their practices and experiences of creating, maintaining, and using personal playlists?

By examining what is important to streaming users when they create and use their playlists, I will shed light on individual user logics, structures, and preferences regarding content creation, organization, and music use in this relatively new digital context. I will first review the existing literature as I begin to construct my analytical framework.

\section{The Literature}

Practices related to streaming services have yet to occupy researchers to any extent, even though the use of music-streaming services continues to grow, especially in the Western world. Referring to the values that reigned in collecting in the pre-digital era can help us begin to contextualize the perspective on collecting music in the digital age. Walter Benjamin's "Unpacking My Library," for example, commemorates the magnificent rituals of the book collector and emphasizes three qualities in particular: ordering - "For what else is this collection but a disorder to which habit has accommodated itself to such an extent that it can appear as order?" (Benjamin 60); owning - "The phenomenon of collecting loses its meaning as it loses its owner" (67); and desiring - "To renew the old world - that is the collector's deepest desire when he is driven to acquire new things" (61).

Jean Baudrillard distinguishes collecting from the inferior activity of accumulating (22), noting that objects have two possibilities: they can be utilized or they can be possessed (8). The first refers to the ways in which people harness things in the interest of asserting practical control in the real world. The second refers to the subjective and social status of the object divested of its utilitarian function and abstracted from any practical control. Its destiny is now to be collected, rather than used (8). Baudrillard further finds that the practices of "true" collecting include pursuing a succession of singular objects, cultivating the passionate abstraction that is called possession, and, of course, seeking out, categorizing, gathering, and disposing of objects themselves $(8-10)$. The collection cannot exist as such without an internal scheme that may speak to others but always, first and foremost, speaks to oneself (22). The collection's value is often individually assessed. Roy Shuker insists that "[t]here is no 'typical' record collector" (237); his study describes significant variation in the given fan's association of recordings with identity formation and life history, accumulation and completism, and discrimination and connoisseurship (237).

Whereas Baudrillard, Benjamin, and Shuker consider the collection of physical things, the present article considers the collection of something that has now apparently surrendered its physical materiality: music. What role the music's material form, the music media's "thingness," plays in the storage, processing, and transmission of information (Straw 233) is of interest. "As key elements in the material culture of music, formats - like the $78 \mathrm{rpm}$ record, vinyl album, and compact disc - were marked by distinctive sizes, storage capacities, and characteristic relationships between musical and nonmusical information" (233). Attending to a 
format's aggregative features can reveal inherent, and telling, differences - or what media theorist Friedrich Kittler (qtd. in Straw 233) would call a format's "storage capacity." For example, an LP's material form carries with it a distinctive protocol for listening, and for encountering a given performer's personality via the information and a deliberately ordered series of tracks (Straw 234). However, with LPs too, audiences have been able to subvert given music structures in personal listening practices, and customization has been cultivated in individual recording practices, e.g. in home taping and cassette mix taping. Nevertheless, digital formats like CDs and, later, MP3 files have made the disruption of artists' "album" presentations easier by enabling the listener to select and reorder at whim (Straw 234). Also, at least since the advent of Napster in 1999, online music practices including music downloads have facilitated mass customization of the musical experience with aggregation of songs independent of artist, genre, etc. (Jones 230) often with emphasis on single songs rather than albums (Jones and Lenhart 194).

This already hints at how the digital format affects the listener in the context of music aggregation. In understanding digital music collecting the user interface of MP3 players and online music services is key, incorporating a range of material infrastructures, processes, and practices (Beer 85). The properties or features of objects, or specific settings available to a given technology, invite particular uses (Ian Hutchby quoted in Gillespie, Boczkowski, and Foot 23). Still, users are always able to find ways around this attempt at interpretive closure so that any given object or piece of technology encompasses a range of intended or unintended uses. The relation between how something is supposed to work and how people actually use it will impact both the argument and the conclusions of the present article. Its detailed look at audience practices will engage both the technology's capacities and the user's capabilities and interests, specifically in light of contemporary conditions for consumption and the individual premises for music listening.

In comparing music-streaming practices with earlier ideals for music collecting (recall Hornby) one immediately encounters the dilemma that digital formats and streaming services make it impossible to "collect" music as such (Burkart 247) because the format offers music through subscription rather than ownership. Symbolic substitutes for physical collections must then arise through software interfaces designed to enable (or restrict) access to music and other cultural objects encoded in digital formats (247). These interfaces require us to cede control to technology in a way that in turn offends the music collector's sensibility: "The controlled life of the music user within the digital enclosure seems incommensurable with the empowered music user who once went to record stores and bought, sold, traded, and collected CDs, LPs, and cassettes, who retained the rights of first sale with which to build a collection" (249).

Nevertheless, as the forthcoming analysis will demonstrate, users continue to covet, collect, stockpile, and enjoy music in these digital formats as though music remained somehow a cultural object, which should be analyzed in relation to artifacts even if they are not artifacts but only software (Sterne $831-32$ ). Digitized music via streaming services and MP3 files, after all, is "designed for massive exchange, casual listening and massive accumulation" (Sterne 838). These qualities have liberated recorded music from the traditional economy of exchange value, within which ownership status is central. 
Relatedly, Marjorie Kibby found that music listeners who are actively engaged with their collections interact with digital files just as they did with physical formats. Digital music files play an important social and symbolic role in their owners' lives, and organizing, classifying, and aggregating them even gives the digital content a kind of materiality (441). While this is likely true regarding CDs and even individual MP3 files, Kibby's study does not encompass streaming services, where the continuity begins to break down, as the owner becomes the renter.

Changes in patterns of human behavior are never technology-led alone. Still, the popularity of music-streaming services manifests a shift in consumer behavior that is about to happen. The music distribution era with linearly programmed channels and objects or units for sale is now moving towards a consumption era where access is valued over ownership (Mulligan). Subscription models are cannibalizing sales of music: in Norway music downloads fell by $21 \%$ and physical sales fell by $29 \%$ from 2012 to 2013 (Dredge).

In this, new fan orientations and alignments with regard to this enterprise encompass hoarding, sharing, and searching activities as a means of creating self- reflective digital music collections (Burkart 248). In cyberspace, that is, people collect lists rather than objects, and those lists serve as a form of personal expression that derives from but also supersedes the record collection (McCourt 251). Eighty-two per cent of all user-generated playlists in WiMP Music have unique names, confirming playlist making as a highly individualized practice (Maasø). As such, music is a complex example of compulsive acquisition because music collections are at once archives and participatory practices (Kibby 428), with users as content producers in relation to the contexts and structures of personal music consumption.

More precisely, online users have become content curators, providing editorial perspectives by highlighting particular content on websites and services that allow them to categorize and organize collections of content created by others (Changtao et al. 659). The concept of curation is a constructive model and metaphor offering a solution to the issue of information overload online (Liu 3). It is based on ad hoc expertise depending on skill sets and/or knowledge of topics or events, and is associated with multiple activities or interactions (i.e. collecting, organizing, preserving, filtering, crafting a story, displaying, and facilitating discussions). In socially distributed networks these activities are often interconnected and feed back into each other (3). Nevertheless, Changtao and colleagues found that a majority of online users view curation as a personal activity, rather than a social one. Online curation then might provide a more personal value to the curator by collecting and highlighting other sets of content than would be offered by using other methods, like search (667).

Digital music archiving hence involves new sets of values in relation to music collecting - the intangibility of digital files makes the music less emotionally valuable than a recording medium you can hold in your hand, Tom McCourt argues (249). Their visual and tactile aspects are reduced to simply data, metadata, and thumbnail images, and in this way they are unable to contain their own histories (250). Paradoxically, this lack of materiality and emotional resonance heightens the listener's interest in sampling, collecting, and trading music in new ways that make these experiences more intense and intimate than owning a physical recording (250). 
This is because digital technology offers more possibilities for modifying, altering, and recontextualizing original content in ways that heighten utility, power, and control for the users (251). Specifically, digital media make people want to compact music in archives, cultivate immediacy in the ability to sort and regroup files effortlessly, and devote attention to customizing this content. "Fluidity, rather than integrity, is the defining characteristic of digital technology" (251).

Digital music technologies now regularly govern the everyday experience of time and space (Bull) in ways that have become normalized as habitual and mundane music practices (Beer 85). The study of these reconfigured music practices is complicated by their inherent complexity and unpredictability, according to Beer (78). It is my hope that the present article will contribute empirically grounded observations regarding the precise implications of music-streaming services for this growing area of inquiry.

\section{Methods}

I will apply several methodological models to my engagement with people's sensemaking regarding their playlists, incorporating stated assumptions and strategies, actual practices, and a range of personal experiences. I began with a self-reported diary study, in the hopes of avoiding the potential distortions associated with retrospective inquiries (Hektner, Schmidt, and Csikszentmihalyi 7).

I recruited heavy streaming users systematically to ensure that this study would capture people with prodigious abilities to use technology as well as a great deal of originality in user practices. Heavy users have had their streaming service subscriptions for at least a year and use it daily (five to seven days per week). I engaged half of the study participants following visits to three high schools in Oslo and Akershus, Norway. After circulating participation proposals to about 60 students, aged 17 to 18 , I left with 16 acceptances, from which I chose six. I engaged the other half of my participants by circulating information about the project on Facebook and Twitter, requesting interested users to contact me. Twenty people, aged 21 to 60, replied, none of whom were known to me previously. Altogether, the final group of 12 participants included five male and seven female Spotify or WiMP Music subscribers and encompassed high school students, advanced degree students, and workers in various positions.

The initial instruction to the participants was to write diary entries on every musiclistening session that involved streaming services during four sampling periods that lasted from two to three days. In the interest of securing reports of an everyday nature, participants were not alerted about the sampling periods in advance-SMS and emails indicated when a period was about to begin and end. Diary entries revolved around seven questions that focused on (1) the listening context (location, date, time); (2) the music context (what music, from which source, why start to listen now, how was the music found); and (3) the listening experience (a description of the use of the music, any parallel activities, the social or personal setting, any distractions, emotions, and so on).

An earlier pilot study revealed that users would likely have different preferences for reporting their streaming experiences, so diary material was allowed to encompass 
handwriting in diary books, text messages, emails, screenshots from personal media devices, and replies in spreadsheets created in Google Docs.

\section{Observation and Interviewing}

To complement the diary descriptions of participants' online behavior, I followed their Facebook profiles during the two months of diary reporting. I also observed their "scrobble" activity via the digital platform Last.fm, a feature that finds, processes, and distributes information about digital music listening. This alternative tracking mechanism allowed me to determine whether behavior patterns changed during the testing periods.

The multiple components of the study's design made individual participant briefings a necessity prior to the investigation, and I managed this via face-to-face conversations with all but one participant (who lived some 600 kilometers away and was briefed by telephone). In these briefings I addressed research topics and ethical concerns such as Facebook friending, Last.fm observation, my frequent inquiries during the upcoming testing period, and the maintenance of anonymity. All participants consented in writing to take part in the study, and the Norwegian Social Science Data Services accepted the project.

The diary study was followed by in-depth semi-structured interviews that lasted between 40 and 60 minutes. Interview guides included a fixed section with standard questions and an individually adjusted section that followed up on the conversation and participant in question. All the participants brought along their most-used devices for streaming music. This helped me to develop detailed insights through precise questions, and helped the participants to speak more freely, because the content and practices in question could be elucidated in direct relation to the actual playlists. All interviews were recorded, transcribed verbatim, and coded in HyperResearch.

Also following the observation period, I continued to monitor the participant accounts in Spotify and WiMP Music and captured screenshots from these interfaces. This proved to be so valuable that, after the study had ended, I asked participants to continue to send me screenshots with overviews from at least one of their streaming devices and received permission to use these as illustrations as well. All of the participants except one sent pictures that helped me to further ground my analysis. In addition, a sporadic email exchange continued for a few months with some of the study participants. I formally ended these relationships by informing everyone that the data gathering was complete, after which I broke the Facebook and Last.fm connections as well.

My accumulation of data over time produced a comprehensive impression of evolving, individualized, and contextualized user practices with regard to the fluctuating circumstances surrounding the music-streaming services. All of the participants turned out to be passionate music fans and were very generous about sharing their experiences. Most wrote relatively detailed daily reflections, sometimes multiple times a day, and this material predictably presented users who were investing more than most in maintaining their music collections. Obviously, less enthusiastic music listeners, or listeners who were less interested in sharing their experiences, would be harder to engage in this kind of investigation. My sources generated rich 
and detailed descriptions of music-streaming practices, from which I derived the following analysis using a bottom-up approach. All participant names and playlist titles presented in the analysis are anonymized.

\section{Findings: Structures and Logics of Personal Playlists}

All of the participating music-streaming users had personal playlists in Spotify or WiMP Music that they described as the playlists they used the most in everyday listening. Within the relevant 12 streaming accounts, the total number of personal playlists varied from one to 100 , and the number of tracks in each playlist varied from a few to more than 1,000 .

During the observation period of the study, ongoing playlist activity was evident. Some participants added and deleted entire playlists frequently; others modified and updated content or titles of existing playlists. Some simply streamed the playlists without changing them much at all. This variation in playlist manipulation indicated that users demonstrate a lot of individuality in how they approach music-streaming services, and further that playlists can be regarded as either closed or open "units" of music, depending upon how static or dynamic they are. In the analysis that follows, I will rely heavily upon these two terms as opposite poles of playlist behavior, though I remain aware that, in fact, most people combine them to different degrees and ends.

\section{Static Structures}

With a static playlist, the user basically retains the original aggregation of music for the life of the list. Tracks ordered within the structure of original album releases represent (and inspire) numerous static playlists, even though the streaming service enables the individual to reinvent the album as he or she sees fit.

Personally created playlists become static when the composition or editing of them stops. Sometimes the playlists are felt to be complete; other times they are forgotten, abandoned, or replaced. A playlist can become static immediately after it is created, or more gradually, as was the case with the playlist Sofia (30) made for her 30th birthday party. She assembled the playlist (titled Gibberish) in the weeks leading up to the party, in collaboration with invited friends from her Spotify network. The playlist remained static after the party, though Sofia still listened to it occasionally (diary notes/ interview, 6 May 2013).

Another typical situation in which playlists turn static arises when "best of " playlists for a given year are abandoned as the next year arrives. This does not imply that users never listen to them anymore but rather that the lists go from active to archival. The aggregation of favorite tracks in "best of " playlists was a common practice for several study participants. Marius (24) felt that music-streaming services made it very easy to summarize a year musically, thanks to their immediacy and responsiveness: "So if I hear a track that is immediately good or I return to [it] several times, I drag it into the list there" (interview, 28 May 2013). 


\section{Dynamic Structures}

As mentioned, dynamic playlists can become static over time, but static playlists can be revived as well. Once-static content, imported from external sources or other selfmade compilations, can supply a resource for a new playlist. Emma (17) consistently used copies of her existing playlists as a basis for new ones in Spotify. She deleted some songs, kept others, and added new ones, so that tracks began to overlap across her playlists.

Truly dynamic playlist management generally implies a steady increase in content; more of the study participants manipulated by adding than by subtracting. Still, for some users, deletion was part of the dynamic playlist process. During my interview with Louise (17), she spontaneously removed a Rihanna song from her playlist because it was too hip hop for her taste: "If I get tired of them [the tracks], I don't like them anymore, so then I just delete them" (interview, 23 May 2013). Louise's frequent playlist updates led her to maintain only one personal playlist in Spotify, titled Star, in addition to a static list featuring her favorite band, Maroon Five. She consistently updates Star according to her latest preferences. Just before I recruited her, she had cleared the playlist of content, because it was a "mess." Yet by the time of my interview with her three months later, Star again had 193 tracks, including "Avril Lavigne, and actually a lot of rock, but also some kind of hip- hop, but now I'm not fond of that hip-hop anymore. And soft rock. And pop in general. And a little bit of old songs and such, like jazz and [ . . . ] it's very mixed, in a way" (interview, 23 May 2013). This static/dynamic list demonstrated her restless relationship with different kinds of music.

\section{Temporary Playlists}

In addition to updating existing playlists, a dynamic approach includes frequently creating new playlists. Nathalie (17) is very "playlist oriented," meaning that most of her listening sessions catered to playlists. She retained some of them permanently, adding to them according to specific strategies that I will present in the next section. She devoted others to certain passing occasions, such as a walk she happened to take, or a particular day at school. Often she deleted the temporary playlists right after the occasion in question had passed - the playlist titled March, which she described in her diary, was gone by the time of her interview in late April.

Nathalie put a lot of effort into sorting and placing tracks in her context-sensitive playlists, and she was accomplished at seeking and securing the music she wanted, fully exploiting the dynamic potential of the music-streaming service. She also benefited from the immediacy of the service, frequently reordering her playlists even as she was listening to them, using the queuing function: "I press play on the track I would like to listen to first, then place the others in line. As I said, I prefer to know what song I can expect up next" (diary note, 7 March 2013). She also created temporary playlists using excerpts from her various permanent playlists:

I always listen to quiet music when I have to focus on important work at school. The Chillout list consists of well over two hundred unique songs. I tend to create temporary lists with selections from the Chillout list with up to ten songs at the most. 
I do this because I like to have full control over what I listen to [ . . ] Right now I feel very tired, unfocused, and stressed. (Diary note, 7 March 2013).

Nathalie's workout playlist provides a conclusive example of her dynamic, temporary, and immediate editing. She found that it was hard to identify good music for exercising, so the tracks on her permanent training list remained relatively static for a long time. However, when she rearranged the order of the songs, the content felt renewed: "I usually modify the set-up on this list, moving tracks up and down depending on what I prefer that day" (interview, 26 April 2013). The ways in which Nathalie tailored her playlists allowed her the sense of control she needed to appreciate the possibilities of streaming services.

\section{Random Plays}

In other words, Nathalie's music-listening preferences and practices do not jibe with streaming-service features that allow for the elements of surprise and randomness. One such feature is called the "radio," and it is typically used in tandem with personal playlists to automatically extend the user's music selection. This feature uses metadata tagged in the tracks to create streams of music related to the given playlist, so it represents a relatively effortless means of exploring new music defined by the "limitations" of a preselected playlist. Some users also find the radio to be helpful in expanding playlists with related music in the very moment of listening to them. Still, this experience can be mixed - sometimes the radio plays songs that users dislike or do not experience as related, demonstrating that algorithmic guesses at musical similarity can differ from personal preferences in this regard.

Some participants used the radio as a way to avoid making active listening choices. Another popular feature along these lines is the "shuffle," which introduces a lesser degree of arbitrariness into the listening experience because it works only with tracks from a predefined playlist, though it will play them at random. Kristoffer (21) said that he was "a heavy user of the shuffle functionality, and very often I let playlists with hundreds and/or thousands of tracks live their own lives with the shuffle" (diary note, 7 March 2013). This form of listening was at once dynamic and controlled for him, because he had personally endorsed the shuffle's options beforehand.

Participants also associated the shuffle with a particular mode of listening. In contrast to manually edited playlists or the prearranged "album mode," the shuffle almost insists upon somewhat distracted or casual listening, because the user cedes control to the software. Yet adjustments can still be made, even in shuffle mode, to fit the "logic" of randomness. Ha kon (17) stated, "The newest release of the band Eluvative is like that [it requires an adjustment]. One track is called 'Epilogue' and one 'Prologue'. . . . It is okay to listen to them through [the first time], but I will instead delete those two tracks from the playlist [so the music suits the shuffle]" (interview, 7 May 2013). Ha kon approached music at the level of independent tracks and found the album format redundant as a context. His playlist practice demonstrates that even shuffle mode and distracted listening require effort on the part of the listener to remain in control despite letting the software direct the flow of the content.

Thus far I have presented various ways of managing playlists at a structural level. The ability to alter the order of, and the arrangements among, playlist tracks enriches the 
user experience by adding either elements of chance or possibilities for greater control. Yet the millions of tracks that are available from music-streaming services provide personal playlists that have been aggregated according to schemes that transcend the structural. People assemble playlists according to "themes, events, experiences, relationships, or as a sort of 'branding' akin to DJ practices for the creators," McCourt states in relation to MP3 files from P2P downloads (251), suggesting that users of flexible, personalized, and mobile streaming applications also find their own ways of managing music in playlists. I will next test this conclusion against the logic informing the personal playlists of streaming users.

\section{Standardized Categories}

Playlists made in accordance with outside systems of music collection and grouping occurred among the study participants. Some people imported self-curated collections from other digital archives, such as iTunes. Others remade playlists with content from their physical record or CD collections, in order to make that music available on their mobile streaming devices

As already noted, the album as such appears to supply a compelling logic for the personal playlists of Spotify users, while users of WiMP Music are offered a feature that allows them to mark album favorites within the platform, foregrounding particular albums in the streaming interface and thereby making album playlists redundant. Spotify did not have this feature during the study period, so playlists were saved in a long list that sometimes only partially displayed the whole title of the playlist. As the number of one's playlists grew, this characteristic made it increasingly difficult to scroll through the selections, particularly because titles (and playlist content) were hard to remember when they were cut off. Spotify has since introduced a feature labeled My Music, which incorporates the capacity to save whole albums and browse music by artist and album, making the platform's content more akin to a physical music collection.

Despite the relative prevalence of sorting tracks by album, streaming users still managed to insert themselves (or a shuffle) into the listening experience: Emma (17) noted, "I never remember track titles, so I just playlist the whole album to find a particular song again. Some of these songs hence recur on several of my other playlists as well, so this [the album playlist] is really just an intermediary step" (interview, 11 June 2013). Another approach related to the "album playlist" is the aggregation of several albums into a longer playlist. This works equally well in either Spotify or WiMP Music and allows the user to sort content by performer while relying upon the ordering of existing albums. Along these lines, Jon (60) had collected all of the singles by a band called Mister Fox, which, as far as he knew, had never been released independently but only on compilations: "If you search Mister Fox, you get some peculiar French rappers and quite a bit of other stuff, so it is very nice to have it [all] gathered [in one playlist]" (interview, 8 May 2013).

Though Jon did not identify himself as a "playlist guy" as such, he did maintain several lists, sorted into different categories, such as all of the recordings of a single composition called "Theme de Yoyo" or the genre theme English jazz. The organizing logics of playlists like these resonate with existing classification categories in popular music as well. Artist, album, composition, and genre are standard 
groupings used to structure tracks as products, along with more esoteric categories such as producer, label, composer, or year of release. When music is aggregated in streaming services, people tend to turn to these sorts of grouping schemes first as they create personal playlists.

\section{Individual Playlists}

Recalling Kittler's concept of storage capacity, the streaming services have aggregative features whereby user participation enables listeners to become content producers of contexts and structures for their music consumption via personal playlists. Included here is the ability to split up existing aggregated structures (like original album releases) according to personal preference, indicating once again that playlist practices often benefit from a high degree of autonomy and individuality.

Participants' playlists demonstrated many schemes that transcended standard classifications. Some favored musical aspects: Ha kon (17) had playlists sorted by the tuning of the tracks, named (Drop D) and other band (Drop C/C). He listened to them when he practiced the bass guitar to help him master the alternate tunings. Nathalie (17) described her playlist Unique as follows: "The voices of the artists stand out from 'the ordinary,' and that is exactly what is so fascinating [about these songs]" (diary note, 7 March 2013). Another of her playlists favored slow songs of gentle character - "The Chillout list is my library for quiet music" (diary note, 7 March 2013) for going to bed or doing schoolwork. Along these lines, Ha kon collected "kind of the most heavy stuff I have" (interview, 7 May 2013) on his playlist titled Roughish-rapid-rhythm-stuff.

Playlists also responded to themes or things outside the music universe, and there was truly no limit to the inventiveness here. Many of the younger participants used TV series and films as references-Nathalie's (17) playlists included Mystic Falls (representing The Vampire Diaries), Upper East Side (Gossip Girl), Oz (Wicked), and Lima to NY (Glee). Jenny (18) had a playlist with various recordings from Les Miserables, including the full- length version of the movie soundtrack but also orchestral and stage versions of her favorites that she had discovered in Spotify (interview, 29 May 2013). Anne (35), a hobby diver, had a playlist dedicated to water-themed music:

So I thought, "Oh, I have to find some fun music," and I decided to gather all [of the tracks I could find] with a fish theme or ocean and sea themes. And that was a lot of fun because it's very random. Many of the composers might never even have seen the sea. So I started collecting it. Nonetheless, I thought a lot of it was crap [...] I add new tracks when I come across something. I have never listened to it, though. That's not why I have it. (Interview, 21 May 2013)

We saw above how streaming technology invites immediate and dynamic handling of playlists, which inspires a certain kind of arrangement. We now see how the sheer abundance of the online archives inspires a different kind of arrangement - more songs within certain categories are always potentially available, and the users can easily enter a "state of collecting" that responds to the true collector's perpetual desire to renew the collection (Benjamin 61). Anne's water playlist, then, is something to have, not something to use, recalling Baudrillard's description of the collector's urge 
to simply possess things. On the other hand, another common practice produces playlists inspired by or dedicated to very specific contexts, where the use trumps the possession, as we will see below.

\section{Context Sensitive Playlists}

Above and beyond their presence on desktop computers, music-streaming services arose as applications designed for mobile devices like smartphones and tablets, meaning that users are able to listen to them in a host of contexts. The conditions and purposes of these contexts therefore often determine the music aggregations that users fit to them. In this case, however, there are very different levels of context sensitivity and exactitude versus flexibility.

Nathalie's (17) dynamic temporary playlists, for example, demonstrated extreme context sensitivity - she made many obvious adjustments in the interests of manipulating the music according to a momentary listening situation or experience of self. Similarly, Louise's (17) constant editing of her single permanent playlist Star was also context sensitive, in that it was frequently updated to track her changing tastes in music. Both users located their motivation in the immediate listening moment, to which they responded through the exploitation of streaming's comprehensive and immediate search and retrieval functions.

A spirit of exploration governs playlists made in the interests of gathering current releases, exemplifying a context sensitivity that is directed at the immediate present. Sofia (35) had a playlist titled New Finery in which she placed tracks that were new to her, as a sort of staging area, before she decided whether they merited inclusion in her various other playlists. That way she never missed anything. Many users reported that they tended to update and manipulate these "main" current-release (or simply current-favorite) playlists more frequently than their theme-based or situational playlists as well.

Other examples of relatively permanent context-sensitive playlists were aggregations based on daily situations or routines. Playlists might be created to fall asleep to (sleep, fleabag), exercise with (make it count, sweating sweetheart, better go working out), study or work alongside (exam, study night, the work list, or kaam kaam ["work work" in Hindi]), or commute with (onthago, drive-by-smiling, homewards).

Social events inspired playlists too, including celebrations of a birthday and the constitution day, a payday gathering with colleagues, dinner parties, or just casual nights out with friends. Nina (27) recalled:

Just before I was to defend my [master's] thesis, I made a playlist in Spotify called Fight Face. I was terribly nervous right before the exam, and therefore I needed some good up-tempo music that I really appreciated to make me "fit for the fight." And it worked out very well and made sure I was less nervous and ready for the exam. (Email from Nina, 14 October 2013).

Even holidays or seasons in life define playlists, titled things like Alternative Christmas, Upcoming Summer, Spring-Like Winter, or Summery Sun 2013. Erik (18) made a playlist to remember music from a cabin trip with his high school theater: "It 
has become, like, that I kind of associate the songs with exactly then" (interview, 16 May 2013).

This demonstrates that playlists made and used frequently during a specific period can become contextual representations in retrospect. Nina (27) tended to listen to only one playlist at a time; in the interview, this supplied her with detailed flashbacks of various playlist-associated periods: "It has been a long time since I've used it, but back then I played it a lot, really a lot! Used it back and forth to work, and at the office [in which] I worked at the time-we had a radio we could plug the iPhone into, so I played it there too, as music to work with" (interview, 12 June 2013). Playlist titles thus serve as personal hooks for the period (and the theme or content) that the playlist represents:

If you wonder about the name of the playlist Fuck Life\&dance4ever, I made the list at the end of spring, when I was so sick and tired of thesis writing, job searches, and obligations in general that I instead felt the need to listen to some music with tempoand dance away. [ . . . ] Away from all the tasks, the stress and such, hee hee:) That list I kept secret in my Spotify account - not because of the tunes chosen, but rather because of the name on the list and how I felt just at that time. (Email from Nina, 31 August 2013).

\section{The Self and Others as Playlist Contexts}

The email excerpt above demonstrates that Nina's personal mood, feelings, and temper informed the music she preferred - she tended to use the self, that is, as her context for making playlists. Such playlists were common among the study participants, but people's uses for them differed.

Figure 1. "To be honest, my music mood is a total mess. Most likely because I'm a bit indifferent at the moment, feeling neither happy nor sad, hence it's hard to define what I am going to listen to. When I am happy I always listen to happy songs. If I am sad or angry I choose rock. The worst thing one can do is listening to sad music when feeling sad. Then you become much more low that you already are" (translation from diary note, Nathalie, 6 April 2013).

Figure 1 is the screenshot of a diary note Nathalie (17) wrote on the tram, reflecting her own process for choosing playlists according to her mood. She was listening to the playlist reality isn't enough because she wanted to feel tougher (diary note, 6 April 2013). Anger management, tender, happytunes, floating fine, and daydreaming (feelgood songs) were other examples of playlists arranged by mood from the study participants. Likewise, the self is the obvious context for specifically biographical playlists. Nathalie's The soundtrack of my life included "songs I would have played if my life were a movie" (diary note, 8 March 2013). This list was highly dynamic, with continually changing content and titles, as she continued to search for herself and undergo introspective identity work.

In the biographical playlist Memory Lane, Anne (35) had gathered tracks representing "strange memories, good memories of a high sentimental value" (email from Anne, 25 August 2013). During the diary period, this playlist was named Guilty Pleasures. In the interview, however, Anne realized that she did not harbor any "guilty" feelings 
about the music or the time: "It's more a list to reminisce, I think" (interview, 21 May 2013). She then renamed it Memory Lane, which captured its function more accurately.

Some participants viewed mood, feelings, temper, memories, or biographical history as the most efficient and practical dictate for sorting music, because these various internal logics served as hooks for expediting the surveying of potential playlist tracks. These lists could be quite personal, like Nina's Fucklife \& Dance4ever, or even intimate and private, or they could be shared through acts of communication and social identity management. For example, Nina shared other playlists in her streaming account. Some were "statement" playlists: Old Danish aggregated performers exclusively from her native country, to demonstrate the quality of Danish music to a colleague who doubted it. Likewise, she shared her Bjo"rk playlist. When she was younger, her interest in Bjo rk made her feel different, because none of her friends liked the artist. As she grew older, the playlist became a statement about herself (interview, 12 June 2013).

\section{Discussion: Fluidity, Curation, and Control}

The range of ways in which the participants explained their personal playlists proves that music-streaming services invite multiple approaches to and uses of digital music. Applying individual strategies including selective uses of service features, the participants provided new and old music to numerous personal playlists. Some mentioned that service-provided suggestions sometimes inspired their music exploration. Still, neither editorial or commercial content highlighted in the interface nor algorithmically provided music suggestions was emphasized as particularly important in the playlist making, underpinning the autonomous music interest among this group of streaming users. Rather, a dialectic between practices, intentions, sensemaking, and experiences in the participants' accounts was crucial in understanding collecting, curating, and listening to music in this format.

\section{Fluidity}

The music-streaming service's capacity to create dynamic playlists allows the listener to conveniently and flexibly assemble music according to either long-term or fleeting preferences. This process can be experimental or calculated, depending on the approach. Streaming's dynamism resonates with its comprehensive and mediumspecific storage capacity and allows for the insertion of new values into the enterprise of the collector, partly aligning the descriptions of McCourt and Sterne in this regard. Streaming's immediacy in navigation and accumulation lets the collector fetishize abundance as well as the attraction of the singular object (Baudrillard $8-10$ ) and juxtapose passing fancy with long- lasting desire (Benjamin 61). This immediacy also helps the collector use, rather than simply possess, the playlist, adding a significant dimension to its meaning.

Music-streaming services encourage almost effortless editing and rearranging of playlist content, including the relatively instantaneous creation and deletion of whole lists. This can result in lively collector practices but does not appear to elevate the streaming experience over the collection of physical recordings in terms of either intensity or intimacy, as McCourt argues in relation to MP3 files (250). Instead, 
people seem to find streaming services to be much more impactful than physical recordings and even MP3 files in their everyday lives. McCourt's (251) assertion that fluidity is a defining characteristic of digital technology resonates with playlist use, even though, as discussed above, some playlists are relatively static. Others evince what I would call great integrity, in the sense that they are extremely coherent with rather than incidental to the context of the present moment or the user's experience of the self, whether they are intended to last forever or not. Playlists imbued with integrity in terms of the listening moment are acknowledged to be more intimate or intense, because they answer to an immediate need or desire. Streaming music is therefore fluid too, but perhaps not in the same way as collecting MP3 files, with regard to elevating integrity too as an experienced characteristic of the technology.

\section{Curation}

Other playlists reflect the urge to accumulate that tends to define the most enthusiastic record collectors. The tendency to make playlists simply to keep them applies to both album-originated lists and self-curated collections like Anne's "water music." The related playlist practices often resemble what Liu calls an archivist approach to curation; finding, collecting, and aggregating content with the goal of pulling together a diverse set of content from different sources (2). In addition Anne's curatorial practice includes elements of storytelling in how she weaves together selected content based on a themed story that makes sense with explanatory text or commentary (3). We might also recall Jon's (60) summary of his Mister Fox music - "It is very nice to have it [all] gathered [in one playlist]"- as a reflection of an interest in collecting itself, rather than listening as such. Jon's classification is archival, yet it also includes an intention to catalog music more easily to retrieve it, which characterizes librarian curatorial activities (3).

We might thus wonder at Burkart's insistence upon the incommensurability of the activities of the music collector and those of the music service user (246). According to my findings, practices of gathering music into streaming playlists regarded as curatorial activity are clearly associated with principles of collecting. Still, Baudrillard might argue that music aggregation via playlists aligns with accumulating rather than collecting, because of the ease of acquisition of music through streaming services.

Related to practices of accumulation, Burkart refers to practices of music hoarding as ways of gathering music in digital archives in order to share it, which he insists is the best way to embed value in collections in intangible formats (247). I have found, however, that hoarding-like playlist practices ought to be distinguished from those of sharing. They make sense independently as curatorial activity inspired by the access to millions of tracks. This curatorial activity becomes meaningful as it includes similar mindsets to those of possessing collectable objects (Baudrillard 8) with the purpose of creating and maintaining playlists, simply to keep. Despite the obvious fetishization of quantity over quality that accompanies "music hoarding," the role of playlist curator remains relevant and important, both online and in one's inner world.

As Baudrillard states, "The objects in our lives, as distinct from the way we make use of them at a given moment, represent something much more, something profoundly related to subjectivity" (7). Though the "true" collector's requirement of object 
singularity even among those objects that are aggregated in succession is lost with digital content that is made to be shared among the masses, the act of aggregation itself activates the collector's originality and the value judgments that inevitably ensue. Caring for and preserving collections through stewardship, to engender longterm maintenance of and access to the collection for posterity's sake, is a preservationist approach to curatorial activity (Liu 3) that corresponds with the idea of hoarding either static or dynamic playlists simply to keep

Curatorial practices also might reflect a digital renter's perspective upon the meaning of owning a collection, recalling Benjamin (67): the practices of creating playlists and then keeping them encompass experiences of exclusivity and subjectivity that bring about, in turn, a felt ownership of the music, or even notions of self-identity reflected through the playlist. Curating playlists hence also has a communicational potential in order to create compelling experiences or evoke responses among others, as demonstrated by Nina's shared Danish music and Bjo r̈k favorites. These were purposefully arranged, verified, and filtered for presentation, almost with "editorial value" in terms of being assessed as relevant, reputable, and hence meaningful to share (Liu 3).

Streaming services enable practices of music aggregation other than those of dynamic music grouping, ungrouping, and regrouping. Curatorial playlist practices evoke classic record or CD collecting, and we see that the abundant archives of the streaming service become the stock from which to collect, rather than the source of countless playlists to apply and then dismiss as moments in life pass by.

\section{Dealing with Technology}

The album format's apparently paradigmatic influence upon the industry, the artists, and the listener indicates its likely future viability despite the digital platform's ability to disrupt it. The well-respected status of the album in particular applies among streaming users with histories of listening to CDs and LPs and users who privilege the comprehensiveness of the album. Hence original album releases will probably continue to constitute certain personal playlists, unless streaming services offer more immediate ways to foreground albums, like Favorites in WiMP Music or My Music in Spotify.

Nevertheless, when a user's playlists become overwhelmingly numerous, streaming services begin to appear inefficient and unmanageable as collection systems. The service's archive function can seem less than optimal when its interface is overwhelmed. This might explain why some streaming users prefer playlists based on their own individual schemes and context-based sorting rather than album- or artistdedicated playlists. Their own titles serve as hooks with regard to labeling the aggregation or its purpose. In turn, we see streaming practices influenced by streaming technology (and, more specifically, that technology's shortcomings), as more people abandon albums for personal lists because they can sort and review them more conveniently. 


\section{Playlist Purposes: Control}

User control appears to be the underlying motivation for all the different ways in which playlists are aggregated and enjoyed, archived or manipulated, and even abandoned. This aspect of control, however, takes a variety of guises.

Playlists, first of all, represent a means of practical organization when one is faced with an endless archive, coupled with the ability to search and "claim" tracks within it. Whatever the motivation and intention regarding a given playlist, the user asks the same sorts of things from the streaming service: ease of use, accessibility of content, and an overview functionality that is effective and comfortable. Playlists become fixed entities in a technology defined, ultimately, by its fluidity.

Sometimes this practical purpose overlaps with playlists used as a means of individualization: control over this content, that is, implies control over the self. Music- streaming services provide the same vast archive and user features as starting points to all subscribers, who then overlay themselves upon the service via their playlist choices. The playlist represents what is unique to the individual in the context of a much larger, generic platform, and it demonstrates the persistence of the collector's uniqueness despite the circumstances.

Control in the context of playlists is also exerted through personal negotiation and expression of identity work. Playlists curated by moods, feelings, memories, or biographical/relational representations help the user experience mastery over the self, whether those lists remain private or not. Identity work can be furthered in particular through the dynamic manipulation of the playlist, within the immediate context the present moment and the experience of the self. Lastly, users can harness more or less context- sensitive playlists to structure or simply accompany daily life; without them, the sheer abundance of music in a streaming service is too overwhelming to manage effectively or "on the run."

In relation to these aspects of control in playlist use, Burkart proposes that digital music environments respond to the listener's interest in self-reflection by supplying seemingly infinite resources toward that end. He continues, "[The] desire to search would become presumably more intense if a music fan's entire database of digital music were accessible through a single software installation on a central computer, or networked computers, and if an exhaustive list of music holdings could be generated from an online catalog" (248). This is precisely the case with music-streaming services, which then, through practices of both dynamic and static playlist management, proceeds to prove Burkart's final point: "From this line of approach, then, online music searching is a form of soul-searching that can relieve the collector's fetishes for packaging, acquisition, and handling of records" (248).

\section{Conclusion}

In this article I have described some of the tendencies of music listeners with regard to their personal playlists in music-streaming services. These accounts encompass practices, purposes, and motivations for making and using playlists, as well as various approaches to music and technology in general. Taken in tandem with the capacities 
of streaming technology, these aspects form the basis for the user experience. The playlist is as unique as the listener behind it.

We have seen that playlist activity via streaming services introduces new practices and habits but also derives from traditional physical collecting. While physical music collecting has often been about the hunt for rare gems, playlist collecting involves imposing one's will (and oneself) upon an intangible realm of endless abundance. But the hunt still motivates some streaming users; especially those who tend to carefully curate playlists rather than continually revisit dynamic ones. Other streaming users are much more interested in the streaming technology's immediacy and fluidity, which can add music to intuitive experiences.

In all, streaming users demonstrate the potential for individualization in consumption, whereby the meaning of a product (in this case, a playlist) can be utterly transformed through the context and manner of its use. Colin Campbell writes, "Such activities as collecting, gifting or stylizing hence could be seen as effectively 'negating' the product's status as commodity" $(26-27)$. The playlist enables ownership of music even in streaming services because it undermines or narrows the impact of the service's shared features and content in the interests of elevating personal music selection above all else. When we ask our playlists to answer for our lives and selves, we transcend the generic platform from whence they came.

Music-streaming services encompass aggregative features that invite participation and enable listeners to perform as content curators of their music consumption. In the format of music-streaming services the participating listener thereby plays a role equally important as the role of the medium in the storage, processing, and transmission of information, recalling Kittler's concept of a format's storage capacity (qtd. in Straw 233). It follows that to be a collector in this format requires active management of music into playlists.

The more we manipulate and actively maintain our playlists, as well, the more valuable and meaningful they become. This study hence aligns with Kibby's (441) observation that ownership of music is intensified among those users who actively engage with their collections by classifying and revisiting personal aggregations. In other words, playlist management is as important as initial playlist creationsometimes the purpose of the practice is its performance alone.

The practices of playlist makers seem as various and complex as those of the record collectors in Shuker's study. Here, as there, rituals and preferences of collecting are culturally important as representatives of interwoven narratives of desire, compulsion, and identification, all informed by a fundamental love of music as well as notions of cultural value (Shuker 328). Media technologies, from this perspective, must be understood as complex socio-material phenomena and products of distinct human and institutional efforts. Furthermore, they serve as sites for the playing out of tensions between, for example, determination and contingency (Gillespie, Boczkowski, and Foot $6-7)$.

In conclusion, I would return to Nick Hornby's question of whether it is wrong to want to be at home with your music collection. My answer would be no, as is proven yet again by the many and various experiences of streaming users with their personal 
playlists. There is a whole world of meaning in one's music, whether physical or digital in nature, and no sign of abatement in our interest in it.

\section{Acknowledgements}

This article is produced in association with the University of Oslo research project titled "Clouds and Concerts" and is funded by the Research Council of Norway under grant number 205265.

\section{Works Cited}

Baudrillard, Jean. "The System of Collecting." 1968. The Cultures of Collecting. Ed. John Elsner and Roger Cardinal. London: Reaktion, 1994. 6 - 24. Print.

Beer, David. "The Iconic Interface and the Veneer of Simplicity: Mp3 Players and the Reconfiguration of Music Collecting and Reproduction Practices in the Digital Age." Information, Communication \& Society 11.1 (2008): 71 - 88. Print.

Benjamin, Walter. "Unpacking My Library." Illuminations: Essays and Reflections, Trans. Harry Zohn Ed. Hannah Arendt. New York: Schocken, 1968. 59 - 68. Print.

Bull, Michael. Sounding out the City: Personal Stereos and the Management of Everyday Life. Oxford: Berg, 2000. Print.

Burkart, Patrick. "Trends in Digital Music Archiving." The Information Society 24.4 (2008): 246 - 250. Print.

Campbell, Colin. "The Craft Consumer: Culture, Craft and Consumption in a Postmodern Society." Journal of Consumer Culture 5.1 (2005): 23 - 42. Print.

Changtao, Zhong, Shah Sunil, Sundaravadivelan Karthik, and Sastry Nishanth. "Sharing the Loves: Understanding the How and Why of Online Content Curation." Proceedings of the 7th International AAAI Conference on Weblogs and Social Media. (ICWSM), 2 - 4 June 2013. Web. 18 Dec. 2014.

Dredge, Stuart. "Streaming Still Storming as Norwegian Music Sales Rise 17\% Yearon-Year.” Musically, 11 July 2013. Web. 18 Dec. 2014.

Gillespie, Tarleton, Pablo J. Boczkowski, and Kirsten A. Foot. Media Technologies: Essays on Communication, Materiality and Society. Cambridge, MA: MIT Press, 2014. Print.

Hektner, Joel M., Jennifer A. Schmidt, and Mihaly Csikszentmihalyi. Experience Sampling Method: Measuring the Quality of Everyday Life. Thousand Oaks, CA: Sage, 2007. Print.

Hornby, Nick. High Fidelity. London: Gollancz, 1995. Print.

Ifpi Norge. "Det norske musikkmarkedet 2014." ifpi Norge, 16 Jan. 2015. Web. 26 Feb. 2015. http:// 
www.ifpi.no/flere-nyheter/item/79-det-norske-musikkmarkedet-2014

Jones, Steve. "Music That Moves: Popular Music, Distribution and Network Technologies." Cultural Studies 16.2 (2002): 213 - 232. Print.

Jones, Steve and Amanda Lenhart. "Music Downloading and Listening: Findings from the Pew Internet and American Life Project." Popular Music and Society 27.2 (2004): 185 - 199. Print.

Kibby, Marjorie. "Collect Yourself." Information, Communication \& Society 12.3 (2009): 428 - 443. Print.

Liu, Sophia B. "The Rise of Curated Crisis Content." Proceedings of the 7th International Information Systems for Crisis Response and Management. (ISCRAM 2010). Seattle, WA, May 2010. Web. 17 Dec. 2014.

Maasø, Arnt. "Music Streaming in Norway: Four Trends, Four Years Later." Paper given at by:Larm Music Conference 2014, Clarion Hotel Royal Christiania, Oslo, 27 Feb. 2014.

McCourt, Tom. "Collecting Music in the Digital Realm." Popular Music and Society 28.2 (2005): 249 - 252. Print.

Mulligan, Mark. "Why the Access Versus Ownership Debate Isn't Going to Resolve Itself Anytime Soon.” Musicindustryblog, 9 Dec. 2011. Web. 17 Dec. 2014.

Shuker, Roy. "Beyond the 'High Fidelity' Stereotype: Defining the (Contemporary) Record Collector.” Popular Music 23.3 (2004): 311 - 330. Print.

Sterne, Jonathan. "The Mp3 as Cultural Artifact." New Media \& Society 8.5 (2006): 825 - 842. Print. Straw, Will. "Music and Material Culture." The Cultural Study of Music: A Critical Introduction. Ed.

Martin Clayton, Trevor Herbert and Richard Middleton. 2nd edn. London and New York: Routledge, 2012. 227 - 236. Print.

TNS Gallup. “InterBuss Q4 2014. Norges største understøkelse om internettvaner.” TNS Gallup Report received 27 Feb. 2015 Email. 